\title{
Revolutionary thoughts on taxonomy: declarations of independence and interdependence
}

\author{
Quentin D. Wheeler
}

\begin{abstract}
International Institute for Species Exploration, Arizona State University, Tempe, Arizona, USA. E-mail: quentin.wheeler@asu.edu
\end{abstract}

"The greatest obstacle to discovery is not ignorance -

ABSTRACT. Credible biological research depends on accurate species identifications, reliable scientific names, and an evolutionary context provided by a phylogeny or phylogenetic classification. The emphasis on such taxonomic services has detracted from the fundamental taxonomic research necessary to create and sustain such knowledge systems. A taxonomic declaration of independence is presented, emphasizing the unique mission, goals, and needs of taxonomists and taxonomic (collection-based) institutions and the non-experimental yet scientifically rigorous epistemology of taxonomy. At the same time taxonomic interdependence is declared. Questions pursued by taxonomists are planetary in scale and can only be answered given international collaboration and coordination in the growth and development of natural history collections and taxonomic hypotheses. Reciprocity of open access is urged between all nations and the taxonomic community creating information. Countries home to species diversity should allow access to taxon experts so that the world's species are discovered and described. In exchange all specimens collected should be in publicly accessible museums and all resultant taxonomic data, information, and knowledge should be openly available to all who can use them. KEY WORDS. Biodiversity; classification; phylogeny; systematics.

Former United States Librarian of Congress and historian Daniel Borstin warned us of the danger of losing knowledge in a fog of information. As enormous quantities of molecular sequence data are generated for species we are in serious danger of losing sight of why we do taxonomy. Identifications of known species using DNA barcoding, production of molecular phenograms based on a few genes, and even sequencing of whole genomes do no more than modestly advance the core of taxonomy as a science or improve taxonomic knowledge in service to others. As it turns out, taxonomy done for its own sake and to its own standards of excellence results in both an advancement of taxonomy's own goals and the highest quality by-products on which other biologists depend: accurate species identifications, predictive conceptual frameworks in which to interpret observations and phenomena, informative scientific names with which to store, retrieve and communicate information.

Some users of taxonomy in their zeal to make species identifiable have overlooked the central aims of the science of taxonomy. Comparative morphology is as central to taxonomic exploration as species diagnoses, cladograms, and formal classifications. Morphological research is an end in its own right. It is not merely a means to an end such as a species identification or cladistic analysis although it has much to contribute to both. The concepts of homology and synapomorphy connected to complex morphological structures remain as fundamental to the theoretical basis of taxonomy as are theories of species and monophyletic groups. Non-taxonomists who are users of taxonomy rightfully expect to be able to identify species and refer to them by a unique name. Beyond insisting on the ability to identify species users should demand that taxonomic information be reliable and not accept inferior substitutions (e.g., Prendini 2005, Wheeler 2005).

Because of the biodiversity crisis and rapidly changing ecosystems we need the results of taxonomic research more than ever. We need a record of earth's species, their attributes, and their distributions both to inform policy and as a reservoir of data for future scientists to study. Unless and until taxonomy is recognized and supported on its own terms its advances, integrity, and the quality of its results will be diluted.

\section{Taxonomic declaration of independence}

Taxonomy is the science of species and their characters, relationships and classification. At its heart is species exploration that does not end with species discovery, description, classification and naming but continually tests "known" species, adding to the completeness and accuracy of their description and revising their classification as necessary. Because taxonomy 
is primarily an evolutionary-historical science based on patterns of character distributions, it is non-experimental yet highly rigorous in its testable assertions about characters, species, and clades. It has a special focus on the study of characters of species, based on concepts of homology and synapomorphy, that at once reveals the status and relationships of species and constitutes a scientific aim in its own right to understand the origin and transformational history of characters (PlatNick 1979).

The best taxonomy is only possible when taxonomy is respected and supported as an independent science. Taxonomy has its own mission, goals, aims and needs distinct from all others in biology. Taxonomy is independent of ecology. Ecology and ecosystem science seek to understand the interactions of species and the functions of complex living systems. Taxonomy studies monophyletic taxa which requires collecting (not sampling, for insights into local abundance is largely irrelevant to taxonomy's questions) in many places to acquire presence/absence information about species and their distributions in ecological and geographic space. Taxonomy is independent of population genetics and studies of processes of speciation. Taxonomy studies patterns - unique combinations of characters defining species as well as synapmorphies defining clades - and is thus complementary to micro-evolution. Taxonomy is independent of molecular genetics; nucleic acids in and of themselves being devoid of historical information, it is only in increasing levels of complexity that information of relevance to taxonomists emerges; taxonomy is not concerned with mechanisms and functions but with a wholly different set of questions that are themselves global in scale (Cracraft 2002).

Taxonomy is an independent science with an elegant hypothesis testing capacity due to its all-or-nothing claims about the world that are among the most rigorously testable of all theories (e.g., Popper 1959, Gaffney 1979, Nelson \& Platnick 1981).

For decades the core goals of taxonomy have been obscured by blurring the boundaries between taxonomy and complementary fields such as population genetics, ecology and molecular genetics. Some taxonomists seem to want or need the approval of such experimental biologists but why should this be so? How many ecologists or molecular biologists seek or feel the need for the approval of taxonomists to validate their work?

The importance of taxonomy to science and society is self evident: It is through taxonomy that we explore and understand the results of billions of years of organic evolution; that we understand what species exist and where so that we can detect and monitor environmental change and increases or decreases in biodiversity; that we develop a language of biodiversity rich and dependable enough to permit us to know and refer to millions of species; that we explore phylogenetic history in all its richness; and that we reveal the wondrous diversity to be discovered through comparative studies of morphology, behavior, development and other complex attributes of living things.

The theories and methods that have been developed in taxonomy over the past two and one-half centuries have made it among the most explicit and rigorous of the biological sciences, yet its historical non-experimental orientation makes it appear suspect to experimentalists who do not understand its epistemology. Taxonomy declares its independence from experimental biology. It does not want or need to be associated with experimentalism in order to demonstrate its importance or achieve its greatest achievements. Forced merger of taxonomy with other disciplines, even when well intentioned, has invariably reduced the prestige of taxonomy and resulted in funds flowing to more familiar experimental areas. Whether confounded with population genetics in the 1940s, tropical ecology in the 1970s, or molecular genetics in the 1990s, history teaches us that taxonomy suffers when it attempts to advance itself through association with an already successful sister science.

As an independent science, taxonomy has an obligation to pursue and strengthen its unique questions, goals, and infrastructure. We can no longer tolerate confusing our clear responsibilities and aims in a bid to gain the support of scientists who see us as a service at most and who do not share our history, traditions, epistemology, or infrastructure needs. We alone bear the enormous responsibility to our fellow biologists and society to explore species and we alone bear the equally enormous responsibility to build comprehensive collections that document declining species diversity for future generations. We cannot live up to these responsibilities unless we insist that taxonomy is supported and practiced on its own terms.

While taxonomy must be seen as independent of experimental branches of biology it must also be recognized that taxonomy pursues knowledge of species and characters that are not constrained by geopolitical boundaries, ecosystems, or arbitrary periods of time. In part because most experimental and environmental sciences need information about species that is limited geographically or ecologically, its seems natural to nontaxonomists to develop collections and identification tools for local or regional floras and faunas. If we are to respond rapidly, efficiently, and effectively to the need to explore species then we must declare also the logical and practical interdependence of taxonomists and their research resources, most particularly collections.

\section{Taxonomic declaration of interdependence}

Taxonomists and taxonomic (collection-based) institutions around the world are inseparably connected through the rigorous demands of taxonomy as a science. Taxonomy is a planetary scale science with the need to compare and study specimens of all species in a monophyletic group without regard to where or when species live or have lived on earth, where specimens were collected, or where specimens are housed in museums and herbaria. In order to secure the highest quality and most reliable taxonomic information it is therefore essential that taxonomists have access to field sites and museums where relevant specimens are located. Taxonomists sharing interests in major taxa must commit themselves to the expan- 
sion and improvement of our knowledge of those taxa, collaborating and making information and conclusions openly available to all who can profit from the knowledge.

No taxonomist can work alone. Good taxonomy assumes access to all species validly described since 1758 . This in turn assumes access to a major library (soon to be openly available to all through the Heritage Biodiversity Library project and other digitization efforts) and to all relevant specimens, particularly type specimens. The latter is, for almost any significantly sized taxon, to suggest access to scores of collections in as many countries. Accessing the most basic historical information and relevant specimens dictates that good taxonomy done around the globe on a major taxon is necessarily inter-dependent.

No nation can know its fauna (or flora) in isolation. Good taxonomy demands that, even for a single species, specimens be collected from all relevant populations and compared, as well as that closely related species be available for comparison too. The best taxonomy is, therefore, by nature international and cooperative science.

In the past international cooperation took place but informally, impersonally, and over multiple generations. As knowledge grew it was deposited in the printed literature and on hand scribed annotations on specimen labels in collections for colleagues in other countries and other generations to study. Because of the biodiversity crisis and rapid environmental changes we no longer have the luxury of working at such a slow pace. We need reliable and comprehensive information and we need it now. This can only be achieved by recognizing the interdependence of taxonomists and museums around the world working on or curating specimens of any particular taxon and by assuring that they collaborate in ways that can expedite species exploration.

Open access to specimens and information is key. Quoting Daniel Borstin again, "Knowledge is not simply another commodity. On the contrary. Knowledge is never used up. It increases by diffusion and grows by dispersion." Taxon experts should be given access to collecting sites wherever specimens can be gathered that reveal new species or characters or refine our understanding of already known species or characters. Countries that limit such access only assure ignorance about their own fauna and flora and that taxonomic information is less reliable than it could be. Intellectual property rights to patentable substances, an exceedingly rare occurrence from taxonomic field work, need to be dealt with in some way that does not impede species exploration. There are at least two obvious components to protection of interests of such countries. First, taxonomists and collectionsbased institutions conducting field work have an ethical responsibility to make all they learn openly accessible to the international taxonomic community and that of course includes the county of origin. This means data, information, specimens, and other associated observations and analyses. As cybertaxonomy advances (e.g., WHEELER 2008) fulfilling this mandate will become increasingly easy through online publications, databases, image archives, and telemicroscopy that makes specimens themselves remotely accessible. Second are international legal agreements that protect the interests of the country of origin in the rare event that a commercially viable substance is discovered. To a nearly complete certainty the interests of every nation are best met through open access to full and reliable taxonomic information. The more complete and reliable our taxonomy the more likely that such rare profitable discoveries will be made. Being able to identify a species with a property or attribute of interest is necessary so that additional material, specimens or observations can be gathered. Further, in a phylogenetic classification it is possible to focus the search for additional species with the same or a better version of whatever it is that is perceived to have value. National protectionism is well intended but has far more negative than positive results. Ignorance is never preferable to knowledge for the interests of any society. In the trade, countries that open their borders to taxonomic exploration will be repaid by open access to billions of facts about millions of species that will ultimately contribute far more to the welfare of that nation state than fencing off its biodiversity.

\section{Conclusions}

"(But) it's better to lose some of the battles in the struggles for your dreams than to be defeated without ever knowing what you're fighting for." - Paulo Coelho

I agree with the Paulo Coelho quotation (above). I would rather struggle with limited resources and do as much species exploring as possible while practicing taxonomy at its highest level of excellence than sell out the integrity of the science in order to get large sums of money that are ultimately of greater value to some other science. I am, however, optimistic and believe that by promoting taxonomy on its own terms the international community of museums and taxonomists can convince society to support its work and infrastructure. It is imperative that taxonomists remind fellow biologists and themselves why taxonomy is important, what makes it a rigorous independent science, and what the costs of unreliable taxonomy will be on the advancement of science and human and environmental welfare. Waiting for sister disciplines to recognize the importance and independence of taxonomy is futile; they lack the perspective and altruism to do so. Attempting to advance taxonomy by attaching it to the missions, goals, priorities and needs of some other branch of biology has failed repeatedly and is an equally futile option. The only option by which taxonomy can reinvigorate itself, and re-conceive and rebuild its infrastructure from collections to cyber tools, is to declare its independence from experimental biology and assert its unique mission, aims and needs.

At the same time taxonomy must exert and make known the interdependence of taxonomists around the world and of the hundreds of museums distributed among the world's nations. Taxonomy asks big questions that are planetary in scale 
and that cannot be answered if artificially constrained by geopolitical borders, ecosystem boundaries, arbitrary periods of historical or geological time. Taxonomy at its core is the science of species and characters and of clades and synapomorphies. In order to fully understand the origin and diversification of characters or the origin and multiplication of species, taxonomy must be pursued on its own terms. Species exploration is not as simple as completing an inventory, it involves also the never ending challenge of comparing, assessing and interpreting the meaning of every newly collected specimen or discovered character. This means as an independent, historical science that makes allor-nothing claims about characters and their distributions, species, and their relative recency of common ancestry.

It has become fashionable to confuse the aims of the science of taxonomy with those of other sciences. For example, phylogenetic biology focuses on so-called phylogenies in the service of nearly every branch of biology except taxonomy, generating stick figures that are never interpreted to improve formal classifications which are the general reference system of HenNig (1966). Phylogeography that violates fundamental elements of the theories of Hennig to wantonly confound tokogeny with phylogeny. Population biology whose "population thinking" so permeated taxonomy as to blur the distinction between historically informative characters and meaningless (at the species level) "traits" (see Davis \& Nixon 1992, Nixon \& WheEler 1992, LucKоw 1995). Tropical ecology that would have taxonomists waste their time (relative to the questions and goals of taxonomy) gathering data on local species abundance in their field work and subjugating the intellectual rigor of taxonomy in the interest of expedited species identifications, even when those identifications are demonstrably inferior to taxonomy done well. Molecular genetics that would have taxonomy neglect one of its primary directives to discover and understand the origin and transformation of morphological characters in deference to a single, historically anemic source of evidence. Considering the number and ferocity of such onslaughts on the integrity of taxonomy it is remarkable that taxonomy continues to exist in a recognizable form at all. Yet it does and its theories and methods have never been better. There is in every generation a percentage of young scientists drawn to the incredible intellectual challenges and rewards of taxonomy, of species exploration, of character analysis, of Linnaean classifications, and of curating collections to mirror the world's species and character diversity. We need only remove the obstacles from taxonomy, fund and encourage its independent practice, enable its logical international and inter-institutional connections, and get out of its way in order to witness the greatest advance in our knowledge of species and characters in history.

We have a unique responsibility to protect taxonomy from confusion with the goals of other fields. This is because no generation that follows ours will have access to so many species or characters. We alone can create a legacy of museum specimens and field observations to enable science and hu- manity to continue their two thousand year old quest to understand what biological diversity at and above the species level means. The stakes are high. The cost of continuing to ignore the unique needs of taxonomy are infinitely greater than any amount of funding we might allocate to taxonomy and taxonomic collections. Taxonomists must cease to be apologists for their own proud science and fellow biologists must cease attempts to recast taxonomy in their sub-discipline's image. And finally, nation states must recognize both the unique contributions their fauna and flora can make to the advance of taxonomy and their dependence upon conclusions drawn from studies of specimens around the globe. Knowledge of species and museum collections are ultimately treasures of humanity and deserve to be built and shared in a completely open way for the benefit of Mankind.

\section{LITERATURE CITED}

Cracraft, J. 2002. The seven great questions of systematic biology: an essential foundation for conservation and the sustainable use of biodiversity. Annals of the Missouri Botanical Garden 89: 127-144.

Davis, J.I. \& K.C. Nixon. 1992. Populations, genetic variation, and the delimitation of phylogenetic species. Systematic Biology 41: 421-435.

GAFFNEY, E. 1979. An introduction to the logic of phylogeny reconstruction, p. 79-111. In: J. CRACRAFT \& N. EldREDGe (Eds). Phylogenetic Analysis and Paleontology. New York, Columbia University Press.

Hennig, W. 1966. Phylogenetic Systematics. Urbana, University of Illinois Press.

LucKow, M. 1995. Species concepts: assumptions, methods, and applications. Systematic Botany 20: 589-605.

Nelson, G. \& N. Platnick. 1981. Systematics and Biogeography: Cladistics and Vicariance. New York, Columbia University Press.

Nixon, K.C. \& Q.D. Wheeler. 1992. Extinction and the origin of species, p. 119-143. In: M.J. Novacek \& Q.D. Wheeler (Eds). Extinction and Phylogeny. New York, Columbia University Press.

Platnick, N.I. 1979. Philosophy and the transformation of cladistics. Systematic Zoology 28: 537-546.

Popper, K. 1959. The Logic of Scientific Discovery. New York, Basic Books.

Prendini, L. 2005. Comments on 'Identifying spiders through DNA barcodes.' Canadian Journal of Zoology 83: 498-504.

Wheeler, Q.D. 2005. Losing the plot: DNA barcodes and taxonomy. Cladistics 21: 405-407.

Wheeler, Q.D. (Ed.). 2008. The New Taxonomy. Boca Raton, Systematics Association Special Publication no. 76, CRC Press.

Submitted: 05.I.2009; Accepted: 23.I.2009.

Editorial responsibility: Walter A.P. Boeger 